\title{
"IMPROVED GEOMETRIC NETWORK MODEL" (IGNM): A NOVEL APPROACH FOR DERIVING CONNECTIVITY GRAPHS FOR INDOOR NAVIGATION
}

\author{
Filippo Mortari ${ }^{\text {a }}$, Sisi Zlatanova ${ }^{\mathrm{b}}$, Liu Liu ${ }^{\mathrm{c}}$, Eliseo Clementini ${ }^{\mathrm{d}}$ \\ ${ }^{a}$ Department of Information Engineering, Computer Science and Mathematics, University of L'Aquila, Via Vetoio, \\ Coppito, 67100 L'Aquila, Italy - filippo.mortari@gmail.com \\ ${ }^{\mathrm{b}}$ GIS Technology, OTB Research Institute for the Built Environment, Delft University of Technology, Jaffalaan 9, \\ 2628 BX Delft, The Netherlands - S.Zlatanova@tudelft.nl \\ ${ }^{\mathrm{c}}$ GIS Technology, OTB Research Institute for the Built Environment, Delft University of Technology, Jaffalaan 9, \\ 2628 BX Delft, The Netherlands - L.Liu-1@tudelft.nl \\ ${ }^{\mathrm{d}}$ Department of Industrial and Information Engineering and Economics, University of L'Aquila, Via G. Gronchi 18, \\ 67100 L’Aquila, Italy - eliseo.clementini@univaq.it
}

KEY WORDS: Indoor navigation, route graphs, Geometric Network Model, Semantic building, path finding, 2D floor plan

\begin{abstract}
:
Over the past few years Personal Navigation Systems have become an established tool for route planning, but they are mainly designed for outdoor environments. Indoor navigation is still a challenging research area for several reasons: positioning is not very accurate, users can freely move between the interior boundaries of buildings, path network construction process may not be easy and straightforward due to complexity of indoor space configurations. Therefore the creation of a good network is essential for deriving overall connectivity of a building and for representing position of objects within the environment. This paper reviews current approaches to automatic derivation of route graphs for indoor navigation and discusses some of their limitations. Then, it introduces a novel algorithmic strategy for extracting a 3D connectivity graph for indoor navigation based on 2D floor plans.
\end{abstract}

\section{INTRODUCTION}

Over the past few years Personal Navigation Systems (PNS) have become an established tool for route planning, but they are mainly designed for outdoor environments. An explanation to this can be deducted by some evident factors: in outdoor environments the positioning problem has been easily fixed thanks to global positioning techniques like GPS and also the acquisition of huge amount of datasets for road network generation has been possible using remote sensing and photogrammetry techniques. Indoor navigation is still a challenging research area for several reasons: positioning is not very accurate, users can freely move between the interior boundaries of buildings, path network construction process may not be easy and straightforward due to complexity of indoor space configurations. Public buildings like shopping malls, airports and concert halls are getting bigger and more complex, so it is likely that even people who are familiar with these environments could possibly find it difficult to reach some specific rooms or places. Generally speaking, a navigation system comprises: 1) the determination of the position of a user, 2 ) the calculation of a best path to some destination (presumably the shortest one, the cheapest one, the fastest one), and 3) guidance along the path (Gillieron et al., 2004). Since best path algorithms quite often refer to graph theory, the creation of a good network and data model is essential for deriving the overall connectivity of a building and for representing position of objects within the environment. The path needs to be computed in such a way to maximize the usability and success rate while minimizing the chance of the user getting lost (Fallah et al., 2013). The derivation of a network is a task that consists in the simplification of the building structure in order to abstract its metric (such as distance, angles) and topological features (such as adjacency, connectedness), which are suitable for indoor route planning.

The majority of the indoor models found in current literature ignore at least one of the following aspects: 1) they ignore architectural characteristics. Number of doors, openings and windows is not taken into consideration in many models. For instance, a room could have multiple doors; therefore different possible paths could be considered for navigation purposes. Knowing that a room has only one door implies that this room may be considered as an endpoint and not as a transfer space. If the same room has a window that window could be used for emergency response and evacuation of the premises. 2) Granularity is too coarse. Most of the models abstract a room as a single node within the network graph, not considering a detailed partition of a room space into different areas. For instance, it is possible to consider a large concert hall, or the main entrance area of an airport: this kind of spaces should be subdivided into smaller units in order to be able to locate/guide the user more precisely. Space subdivision has the other positive implication of a more accurate resolution for subject/object tracking within the indoor environment. 3) They are not suitable for generating route instructions that may be converted in natural, human-understandable language. 4) Obstacles and dynamic changes are ignored. This kind of constraints for route planning most of the time is not taken into consideration, due to the fact that the majority of the frameworks for indoor navigation relies on CAD / arbitrary-shaped floor plans as originating datasets. In order to encompass these aspects it is necessary to compute navigation routes dynamically.

This requires taking into consideration 3D semantically-rich building models like CityGML or IFC. 
The CityGML model might provide the topographic space of the indoor environment. Furthermore it presents geometric and topological relationships and certain semantics of interiors like openings, installations, and surfaces. It can potentially provide some of the necessary information for this kind of applications. Gröger et al. (2005) proved that this semantic Information Model is suitable for various applications and showed that connectivity properties between spaces can be retrieved for pedestrian access.

A novel algorithmic strategy for automatically extracting the socalled "geometric network" graph (a routing graph that holds both metric and topological information) for indoor navigation is proposed in this paper. The aim of the work is to overcome the drawbacks and limitations of current approaches found in literature.

Following this introduction, Section 2 illustrates related work regarding the existing approaches to the problem of network generation within the indoor navigation context. Section 3 presents the proposed algorithm that leads to the construction of the so-called "Improved Geometric Network Model" (IGNM). In Section 4 some tests and benchmarks with concurrent approaches are outlined, whereas Section 5 concludes this paper and discusses possible developments left to future work.

\section{RELATED WORK}

Substantial amount of work has already been done in the context of indoor navigation. In this section we provide an overview of current developments on path planning and network extraction for indoor navigation, pointing out their major deficiencies.

As shown in Afyouni et al. (2013), regarding indoor modelling approaches, two main classes are inferred: symbolic and geometric spatial models. The last ones model space as continuous or discrete and basically comprise cell-based or boundary-based representations and they rely on metrics and angles ("the distance from place $x$ to place $y$ is z meters"), while the first ones model space using topological relationships, graphs by capturing the connectivity and reachability between spatial units ("the place $x$ is adjacent to place $y$ "). On the other hand hybrid models try to combine both of the aforementioned qualitative (topology) and quantitative (geometry) aspects and are of interest for the scope of this paper. Geometric models can efficiently integrate metric properties to provide highly accurate location and distance information (necessary elements in most of context-aware applications), whereas topological models maintain a more abstract view of space by providing users with easily recognizable information and by materializing more complex relationships between entities (Afyouni et al., 2013).

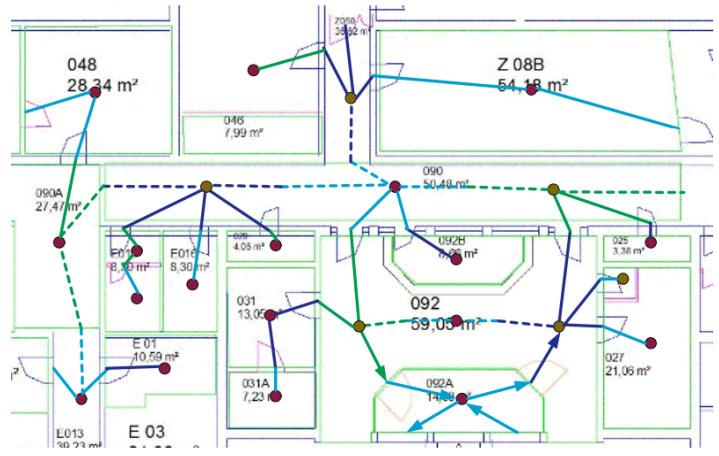

Figure 1. Cell centres and paths overlaid with a floor Plan (Stoffel et al., 2006)

A hybrid spatial model for indoor environments, which consist of hierarchically, structured paths and optional semantic information, is presented by (Stoffel et al. 2006). For buildings with simple rooms and corridors, indoor space (2D floor plan) is decomposed into cells to build a graph structure (Figure 1). They propose a direct mapping for small building instances like small rooms and corridors to the nodes in the graph. The model is hybrid in the sense that nodes and edges can be labelled with qualitative as well as quantitative information. For more complex spaces they propose decomposing cells into several non-overlapping disjoint cells. The major drawback of this approach is that the routes generated by these models are circuitous because they always lead users to the center points of the rooms and cell decomposition is not a fully automated process.

(Yuan and Schneider, 2010) propose an indoor model that produces optimal non-circuitous routes. They consider building parts as cells and classify them into simple cells, complex cells, and open cells, connectors. In their model doors are mapped to nodes and rooms are mapped to edges, with the assumption that doors are the destination of users and furthermore, this allows the construction of length-dependent routes. Moreover, a methodology for connecting doors in concave-shaped rooms is described

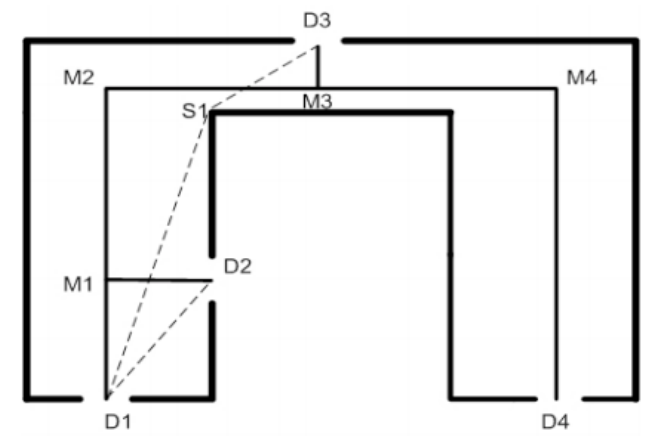

Figure 2. Comparison of S-MAT and door-to-door route (Liu \& Zlatanova, 2011)

In (Goetz et al., 2011) a model which represents indoor environments with topologic, semantic and metric information that allows nearly length-optimal routing in complex building structures (2D floor plan) is presented. The authors focus also on relevant parts of a complex indoor environment which are needed for network construction like corridors and stairs. Additionally they consider obstacles and special semantic areas inside rooms and how to integrate those areas in the routing graph. But there is no description on how to displace the nodes and how to automatically derive the network graph. Apparently the process seems to be not automatic.

Researchers in robotics field have done considerable work using computational geometry algorithms to derive topology networkbased maps for robot motion planning in indoor environments.

Two of the major contributions for indoor path calculation that lead to the generation of these networks are Medial Axis Transform (Blum, 1967) and Visibility Graph (De Berg et al., 2000). The former is a skeleton-abstraction algorithm which can abstract linear features from simple polygons whereas the latter is a graph of intervisible locations, typically for a set of points and obstacles in Euclidean plane. Each node in the graph 
represents a point location, and each edge represents a visible connection between them. These two different yet complementary approaches have been extensively used also in human (pedestrian) indoor navigation since they lead to automatic derivation of networks from 2D topographic features of buildings (such as CAD files or 2D floorplans).

(Liu \& Zlatanova, 2011) introduced the so-called "door-to-door path finding approach for indoor navigation" based on Visibility Graph (Figure 2). It consists in an algorithm applied to 2D floor plan of buildings with complex indoor structure, working on two routing levels, one to get coarse route between rooms, and the other applied to single rooms to acquire the detailed route. Doors (or openings) are approximated with nodes and the rooms with edges. This is in contrast to most currently available network models that treat doors (or openings) as edges connecting rooms (nodes). Each node in the graph represents a point location, and each edge represents a visible connection between them. This approach suffers from two problems: the number of edges increases rapidly, since in convex spaces with no obstacles the number of possible combinations is quadratic, and the fact that consequent route descriptions may result very complex to be interpreted by humans. We find evident difficulties in following instructions as no clear indications can be derived. Instructions look like "the next door is at an angle of 134 degrees and a distance of 9.2 metres".

Lee (2001) introduced a topological data model called Node Relation Structure, which is a dual graph representing connectivity relationship between 3D objects. This representation relies on Poincaré Duality (Munkres, 1984, Corbett, 1985): 3D solids in primal space (i.e. topogaphic space) are mapped into vertices in dual space (logical network), and linking surfaces between solid objects in primal spaces (e.g. doors) are mapped into connecting edges in dual space. In order to abstract building features more accurately, Lee (2004) extended NRS to the so called "Geometrical Network Model", which is a connectivity graph (topological model) plus geometric information such as coordinates of the nodes and link lengths for cost (the shortest/optimum path) computation. How to technically construct the 3D geometrical network model is described in (Eppstein \& Erickson, 1999, Choi \& Lee, 2009): a skeleton-abstraction algorithm formally named Straight-Medial Axis Transformation (S-MAT) is proposed, and consists in a simplified version of medial-axis transformation, capable of providing geospatial analysis for urban environments. A centerline approach results to be a good representation of natural human behaviour within indoor environment. Valid paths are represented concisely, especially in presence of obstacles, though, S-MAT may not represent accessibility within buildings accurately. For instance, it may create weird networks for corridors and fails for large arbitrary shaped spaces. As the space gets wider, a detour is generated, paths are distorted towards the middle of the open space. Hence the medial axis in those cases does not represent a typical path taken by a person. It is evident that these geometrical paths are not suitable for direct translation into route directions.

As it has been remarked, these major approaches have their own benefits and peculiarities. Besides the valuable merits some limitations have been illustrated. We would like to introduce a novel approach, which aims to encompass the positive features of both S-MAT and Door-To-Door on one hand, on the other hand tries to overcome the problems these two strategies present. We refer to those two algorithms as a basis for comparison for their solid popularity in indoor geospatial analysis.

\section{IMPROVED GEOMETRIC NETWORK MODEL (IGNM) APPROACH}

Improved Geometric Network Model consists in a "dimensionally weighted topology network of connected spaces in indoor environments so as to accurately represent indoor route lengths" (Taneja et al, 2011), and it adopts a centreline approach, since space centrelines are an appropriate abstraction for indoor navigation networks as they result to be a good representation of natural human behaviour within indoor environment. As highlighted in Taneja et al. (2011), the fact that visibility-based navigation networks cannot be easily utilized for correcting position data from positioning systems renders these networks less useful for navigation assistance. Moreover, as it will be discussed later, as S-MAT and "Door-to-Door", the approach is $2 \mathrm{D}$-geometry based. In fact, it considers $2 \mathrm{D}$ floor plans as originating data sources, but it produces a 3D network that abstracts floors at different height levels.

The algorithm is based on the assumption that a semanticgeometric model of the buildings is known, as well as topological relationships among spatial entities (such as connectivity between rooms through shared thematic objects such as doors or openings).

The proposed solution consists in a network-generation strategy organized in a hierarchical two-level way, similarly to the approach proposed in (Liu \& Zlatanova, 2011):

- firstly a coarse-grained research can be implemented: this task involves the construction of a weighted graph that only encompasses inter-space connectivity. In other words we are referring to the aforementioned Node Relation Structure. If the semantic Building Model holds geometric information, this task can be performed readily and fast: as suggested in (Becker et al., 2009) ISO 19107 primitives like "representativePoint()" and "centroid()" can be used for abstracting topographic space; edges can link those aforementioned nodes if and only if an opening like a door connects the spaces. Thus, edges in dual representation are placed between adjacent spaces, and weights are calculated as the distance between space centroids. Once the weighted graph is constructed, a shortest path algorithm can be launched on top of it to determine an optimal sequence of spaces between a source and a target (Figure 3).

- once the inter-space path has been found, intra-space path (i.e. physical path) has to be computed. How to compute intra-space path will be described in detail in the next subsection.

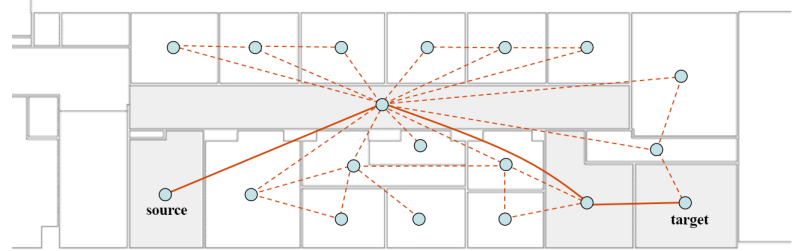

Figure 3. Inter-space path between a source and a target (image taken from Whiting, E. J. (2006))

\subsection{Inter-space path computation}

In this section the algorithm that has been designed in order to construct the physical path will be presented. The strategy is local, in the sense that path construction takes place incrementally room by room along the sequence of spaces 
previously identified by the first step of the proposed solution. The algorithm is composed of several $2 \mathrm{D}$ geometric operations that will be illustrated one by one. Even though the approach is 2D based, the generated network graph will result in a 3D hierarchical network structure like the one proposed in (Lee, 2004). For clarity, we'll refer to what happens only in one room, since this procedure will be iteratively repeated for the other rooms composing the inter-space optimal sequence.

Step 1: inward offsetting. The first geometric operation to be computed on the $2 \mathrm{D}$ polygon that represents the footprints of the room currently inspected is the inward offsetting as it has been described in (Cacciola, 2003) (Figure 4). This operation is essential in detecting large, open spaces that are clearly the weak spot of Medial Axis Transform and will help in overcoming the aforementioned problem of the distortion of the path towards the middle of the open space. Recall that there can exist a set of 0,1 or more inward offset polygons, depending on space configuration. The parameter $t>0$ that defines the Euclidean distance at which the inset polygon will be constructed can be tuned according to some user-defined criteria. As mentioned in (Goetz \& Zipf, 2011), from a geometric point of view, corridors are a special type of rectangular rooms in which two opposing sides are short (i.e., just a few meters) and the other two sides are way much longer (i.e., shortSide $<<$ longSide). A good compromise for parameter $t$ is the lenght of the short side of the corridor, because it generates a sort of virtual navigable corridors around the inset polygon itself. As a consequence, natural human movement is preserved in the proximity of the boundaries of a large space. Unfortunately this value, except from the trivial case of a rectangularly shaped corridor, cannot be extracted automatically and has to be manually assigned as an input value of the algorithm. A good choice of the parameter $t$ is essential.
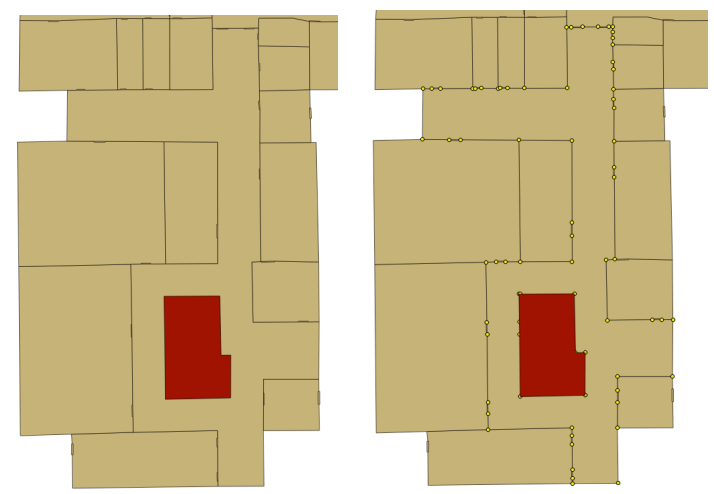

Figure 4. Inward offsetting \& sampling of the contour points

Step 2: sampling of the boundary of the polygons. Once inward offsetting has been computed, a boundary sampling process of the originating polygon can be implemented. This process is legitimized by the fact that we want to encompass semantic objects like doors, windows or more generally speaking openings. As it will be displayed in the next step, this task is fundamental because it forces the Constrained Delaunay Triangulation (CDT) to take into account the portal positions. In fact if both starting end ending points of a door frame are counted, this will result in generating a facet whose constrained edge is spanning over the full width of the door frame itself.

Step 3: computing a Constrained Delaunay Triangulation. Reached this point, a CDT can be computed. The CDT takes as input the point-set obtained in the previous step. The closed chain of edges defining the polygons is meant to be the constraint of the triangulation (Figure 5). As it will emerge later, CDT induces the construction of a centerline network that is a very close approximation of medial axis (Joan-Arinyo et al, 1997). Nevertheless, the introduction of the inward offset polygons can overcome the problems that MAT generates in large open spaces.
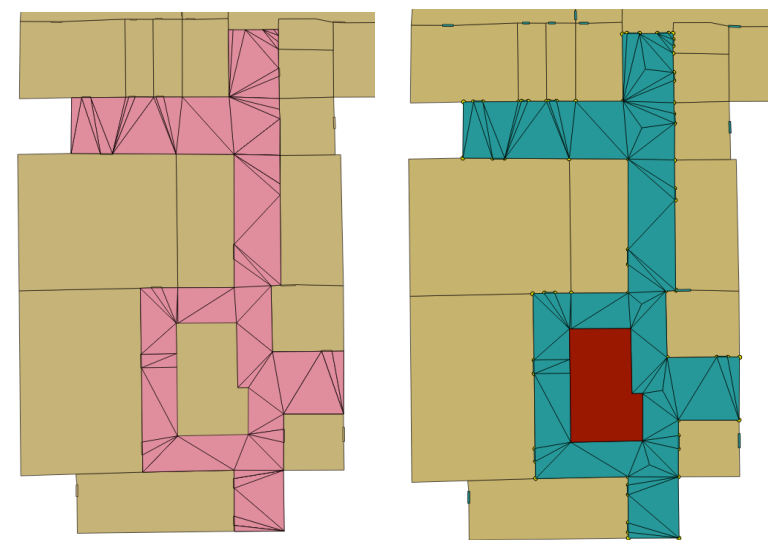

Figure 5. CDT \& subspacing facets with no constrained edges

Step 4: subspace facets that have no constrained edges. After computing CDT and having limited it to the domain identified by polygons, an additional geometric operation has been performed: each facet that has no constrained edges has been further subdivided into three smaller triangles. The subdivision has been made by placing an additional point located on the centroid of the facet named "Crossing" since, as it is visible it represents a space in which possible route alternatives occur. This operation has been adopted in order to suppress unwanted detours on the path.

Step 5: node displacement. Finally, nodes that represent topographic space in dual representation can be assigned to the spaces they belong to. Points are placed at the midpoint of all contour edges of the generated triangles that fall inside facets with no constrained edges. For facets having only one constrained edge, a node is placed on the midpoint of the segment connecting the edges passing through their relative midpoints. A node is also assigned to the space that appeals to the polygon generated by inward offsetting. Regarding the latter, two mutually exclusive conditions may occur:

- The centroid of the polygon falls inside the contour

- The centroid of the polygon falls outside the contour

For the first case, the node can be positioned in the centroid. For the second case, a convex decomposition has to be computed and for each partition a node is assigned, located in the centroid of the partition itself (Figure 6). Furthermore, each node has been marked with particular semantics, based on the type of the topographic space it represents: it might be a DoorAdjacentNode, a DeadEndNode (a node representing a facet with two constrained edges, i.e. walls), a HoleAdjacentNode (a node whose topographic space touches the contour of the inset polygon), Crossing (a node whose topographic space - facet - has no constrained edge), and an ObstacleAdjacentNode. 

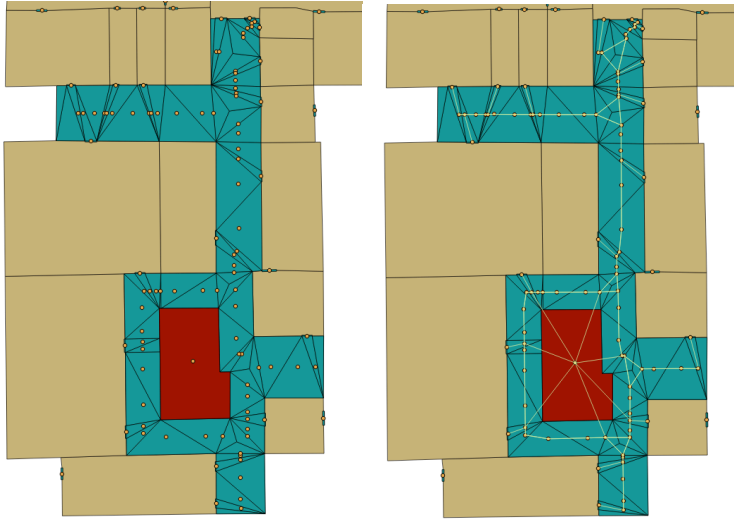

Figure 6. Nodes and edges displacement

Step 6: link nodes. The previous steps contributed to identify and displace the nodes that will populate the Improved Geometric Network graph. Hence, the last operation consists in linking the nodes to each other based on adjacency of the topographic space they represent in primal space. This process has its own peculiarities: DeadEndNodes are skipped since no one wants to navigate to the corner of a room; moreover, some specific nodes (DoorAdjacentNodes and Crossings that are also HoleAdjacentNodes) have been linked to the node(s) representing inset polygons. As visible from Figure 7, the introduced inset polygon determines a "door-to-door" alike network that connects intervisible locations. Edges of the dual graph represent adjacency and connectivity relationships between the cells of topographic space. The graph is not only topological but also geometric: nodes have been geo-referenced in a $3 \mathrm{D}$ context, whilst edges contain metric information (i.e. Euclidean distance between two nodes).

\section{TESTS}

Tests have been conducted in order to evaluate the quality of the proposed solution and to validate the given results. Intra-space connectivity algorithm proved to work correctly in:

- arbitrarily-shaped rooms with concave corners (i.e. irregular concave polygons);

- $\quad$ rooms with obstacles (obstacles have been represented as $2 \mathrm{D}$ geometry in floor plan; the algorithm handles obstacles in the same way as inset polygons, though considering that no centroids have to be assigned to this kind of polygons);

- rooms with open spaces such that convex inset polygons are generated;

- rooms with open spaces such that concave inset polygons are generated (only concave inset polygons that have their centroid inside the contour have been tested);

The floorplans used during tests have been designed with JOSM Java OpenStreetMap Editor and represent OTB Building (TUDelft, The Netherlands). Some benchmarks have been done with Door-to-Door approach. Figure 7 illustrates the difference in the created networks for one corridor.

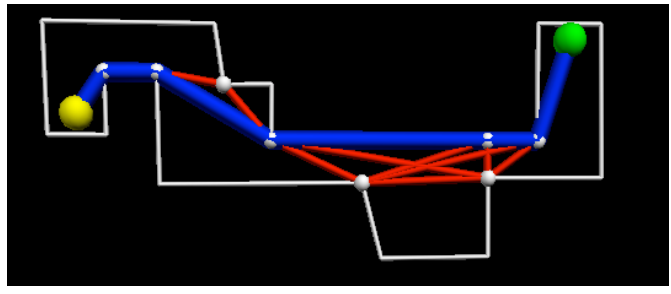

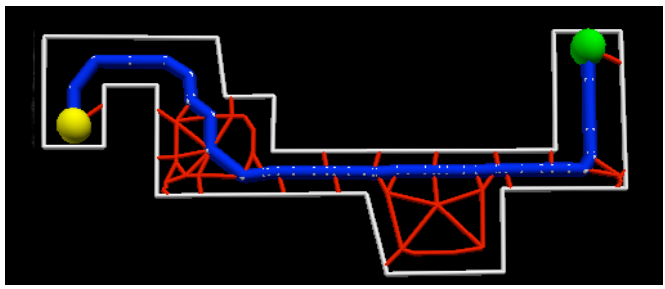

Figure 7. Best path comparison between Door-to-door and IGNM

In average case, IGNM proves to be $\approx 1.6$ longer than the Doorto-Door, which is known to be the optimal-length path. The amount of nodes produced with IGNM approach is higher than Door-to-Door $(\approx 2.3$ more $)$ but the amount of edges is significantly lower $(\approx 0.70$ times $)$. Having more nodes means that indoor space is abstracted with a fine-grained resolution, which is suitable for localization. Since in Visibility-driven approaches the intermediate points of a path between a source and a target are only the concave corners, the tracking of a subject or object is not efficient, with the negative implication that those networks cannot be easily utilized for correcting position data from positioning systems. Hence, the increased number of nodes has not to be considered as a real drawback. Instead, effort has been made in order to reduce the amount of edges which, as previously remarked, grow quadratically with respect to nodes in Door-to-Door approach.

\section{FINAL REMARKS \& FUTURE WORK}

Improved Geometric Network Model proved to be a valid alternative to S-MAT and Door-to-Door. In fact the graph is constructed with a two-level hierarchical strategy, which aims to increase performance, avoiding the geometric computation on unnecessary spaces that for sure will be not touched by the resulting navigation path from a source to a destination target. Geometric algorithm is local, so it is flexible and efficient, and moreover capable of supporting dynamic changes. Differently from S-MAT and Door-to-Door the transformations between primal space and dual space are bidirectional: after graph construction nodes are still aware of the topographic space they belong to. The latter property, plus contour sampling which regularizes the triangulation, contributes to improving the accuracy of localization inside a building. The Constrained Delaunay Triangulation helps in generating a centerline based path which results to be a good representation of natural human behaviour within indoor environment. Nevertheless, as illustrated in Kallmann (2005), Constrained Delaunay Triangulation is an efficient tool for obstacle-avoidance path planning. Obstacles not only are encompassed, but are allowed to be inserted, removed or displaced in the CDT as required during run-time. Inward Offsetting, by detecting large, open spaces that are clearly the weak spot of Medial Axis Transform helps in overcoming the aforementioned problem of the distortion of the path towards the middle of the open space. Moreover, by adding extra nodes inside the generated inset polygon, and by linking them to their nearest DoorAdjacentNodes and Crossings that are also HoleAdjacentNodes, the network somehow tries to reproduce the positive aspects of a "Door-to-Door" navigation between intervisible locations. Summing up: centerline approach for narrow corridors, "Door-to-Door" for larger, open, environments.

Though, some drawbacks are present. For doors located in the corners of the rooms the algorithm might produce some 
serpentine routes, as displayed in Figure 8. This is basically due to the shape of the triangulation, as the facets adjacent to doors are stretched with angles $>90^{\circ}$. In order to overcome this deficiency, some path simplification process might be applied, following, for instance, the method proposed in Agrawala et al. (2001), being careful that removal of nodes doesn't produce unwanted intersections of the path with space boundaries. Let the reader recall that this might be a post-processing step that might result useful only for visualization and / or for generating route directions. In fact we still want to preserve the bidirectionality between primal space and dual space.
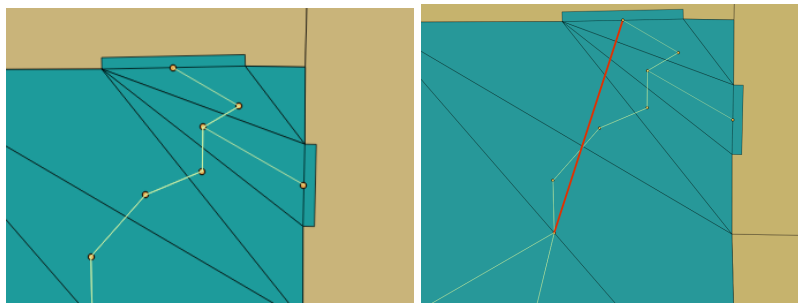

Figure 8. Serpentine routes in proximity of corners and path simplification

Another limitation consists in the technique for displacing one or additional nodes inside the inset polygon in case its centroid falls outside its contour. A non-convex object might have a centroid that is outside the figure. Usually, even though inward offsetting computation might produce non-convex polygons, those latter have regular shapes because building spaces themselves are usually regular. In case the centroid falls outside, the proposed technique of computing a convex decomposition and then displacing nodes in the centroids of the newly generated partitions might result too naive, since it may produce circuitous routes/detours. More investigation about this topic has to be done and is left to future work. Other future developments involve the employment of semantically rich Building Models like CityGML and IFC in order to investigate 3D scenarios and prove the flexibility / extensibility of IGNM to 3D domain. Nevertheless, further experimental investigations are needed in order to better estimate the behaviour of the algorithm with respect to S-MAT and Door-to-Door.

\section{REFERENCES}

Afyouni, I., Ray, C., \& Claramunt, C. (2013). Spatial models for context-aware indoor navigation systems: A survey. Journal of Spatial Information Science, (4), 85-123.

Agrawala, M., \& Stolte, C. (2001, August). Rendering effective route maps: improving usability through generalization. In Proceedings of the 28th annual conference on Computer graphics and interactive techniques, (pp. 241-249). ACM.

Blum, H. (1967). A transformation for extracting new descriptors of shape. Models for the perception of speech and visual form, 19(5), 362-380.

Cacciola, F. (2003). A survey of polygon offseting strategies. http://fcacciola.50webs.com/Offseting\%20Methods.htm"

Choi, J. \& Lee, J. (2009). 3D Geo-Information Sciences. Springer, Berlin, pp. 283-299.

Corbett, J. P. (1985). A general topological model for spatial reference. In: Report of Spatially Oriented Referencing Systems Association (SORSA) Workshop, pp. 9-24.
De Berg, M., Van Kreveld, M., Overmars, M., \& Schwarzkopf, O. C. (2000). Chapter 15: Visibility Graphs. Computational geometry (2nd ed.). Springer Berlin Heidelberg.

Eppstein, D. \& Erickson, J. (1999). Raising Roofs, Crashing Cycles \& Playing Pool: Applications of a Data Structure for Finding Pairwise Interactions. In: 14th Annual ACM Symposium on Computational Geometry, Minneapolis, USA, pp. 58-67.

Fallah, N., Apostolopoulos, I., Bekris, K., \& Folmer, E. (2013). Indoor Human Navigation Systems: A Survey. Interacting with Computers, 25(1), 21-33.

Gillieron, P. Y., Büchel, D., Spassov, I. \& Merminod, B., (2004). Indoor Navigation Performance Analysis. In: Proceedings of the 8th European Navigation Conference GNSS 2004, Rotterdam, The Netherlands.

Goetz, M., \& Zipf, A. (2011). Formal definition of a useradaptive and length-optimal routing graph for complex indoor environments. Geo-Spatial Information Science, 14(2), 119128.

Gröger, G. \& Plümer, L., (2005). CityGML - Interoperable Access to 3D City Models. In: Proceedings of the Int. Symposium on Geo-information for Disaster Management, Delft, The Netherlands, pp. 883-898.

Joan-Arinyo, R., Pérez-Vidal, L., \& Gargallo-Monllau, E. (1997). An adaptive algorithm to compute the medial axis transform of 2-d polygonal domains. In CAD Systems Development, pp. 283-298. Springer Berlin Heidelberg.

Kallmann, M. (2005). Path planning in triangulations. In Proceedings of the IJCAI workshop on reasoning, representation, and learning in computer games ,pp. 49-54.

Lee, J. (2001). 3D data model for representing topological relations of urban features. In Proceedings of the 21st Annual ESRI International User Conference, San Diego, CA, USA.

Lee, J. (2004). A spatial access-oriented implementation of a 3D GIS topological data model for urban entities. InGeoInformatica, 8(3), 237-264.

Liu, L., \& Zlatanova, S. (2011). A" door-to-door" Path-finding Approach for Indoor Navigation. In Proceedings of GeoInformation For Disaster Management Conference 2011, pp. 3-8.

Munkres, J. R. (1984). Elements of algebraic topology (Vol. 2). Reading: Addison-Wesley.

Stoffel, E. P., Lorenz, B., \& Ohlbach, H. J. (2007). Towards a semantic spatial model for pedestrian indoor navigation. In Advances in Conceptual Modeling-Foundations and Applications, pp. 328-337. Springer Berlin Heidelberg.

Taneja, S., Akinci, B., Garrett, J. H., Soibelman, L., \& East, B. (2011). Transforming IFC-Based Building Layout Information into a Geometric Topology Network for Indoor Navigation Assistance. ASCE.

Whiting, E. J. (2006). Geometric, topological \& semantic analysis of multi-building floor plan data (Doctoral dissertation, Massachusetts Institute of Technology). 
ISPRS Annals of the Photogrammetry, Remote Sensing and Spatial Information Sciences, Volume II-4, 2014 ISPRS Technical Commission IV Symposium, 14 - 16 May 2014, Suzhou, China

Yuan, W., \& Schneider, M. (2010). Inav: An indoor navigation model supporting length-dependent optimal routing. In Geospatial Thinking, pp. 299-313. Springer Berlin Heidelberg. 